\title{
The interaction of Thrombospondins with extracellular matrix proteins
}

\author{
Kemin Tan • Jack Lawler
}

Received: 17 July 2009 / Accepted: 30 September 2009/Published online: 16 October 2009

(C) The Author(s) 2009. This article is published with open access at Springerlink.com

\begin{abstract}
The thrombospondins (TSPs) are a family of five matricellular proteins that appear to function as adapter molecules to guide extracellular matrix synthesis and tissue remodeling in a variety of normal and disease settings. Various TSPs have been shown to bind to fibronectin, laminin, matrilins, collagens and other extracellular matrix (ECM) proteins. The importance of TSP-1 in this context is underscored by the fact that it is rapidly deposited at the sites of tissue damage by platelets. An association of TSPs with collagens has been known for over 25 years. The observation that the disruption of the TSP-2 gene in mice leads to collagen fibril abnormalities provided important in vivo evidence that these interactions are physiologically important. Recent biochemical studies have shown that TSP-5 promotes collagen fibril assembly and structural studies suggest that TSPs may interact with collagens through a highly conserved potential metal ion dependent adhesion site (MIDAS). These interactions are critical for normal tissue homeostasis, tumor progression and the etiology of skeletal dysplasias.
\end{abstract}

\footnotetext{
K. Tan

The Midwest Center for Structural Genomics and Structural

Biology Center, Biosciences Division,

Argonne National Laboratory,

Argonne, IL, USA

J. Lawler $(\bowtie)$

Division of Experimental Pathology, Department of Pathology,

Beth Israel Deaconess Medical Center,

330 Brookline Ave., EC/CLS-503,

Boston, MA 02215, USA

e-mail: jlawler@bidmc.harvard.edu

J. Lawler

Harvard Medical School,

Boston, MA, USA
}

Keywords Thrombospondin · Matricellular · Extracellular matrix $\cdot$ Cartilage oligomeric matrix protein

$\begin{array}{ll}\text { Abbreviations } \\ \text { TSP } & \text { thrombospondin } \\ \text { ECM } & \text { extracellular matrix } \\ \text { MIDAS } & \text { metal ion dependent adhesion site } \\ \text { ADMIDAS } & \text { adjacent to MIDAS } \\ \text { PG } & \text { proteoglycan } \\ \text { GAG } & \text { glycosaminoglycan } \\ \text { MMP } & \text { matrix metalloproteinase } \\ \text { LRP } & \text { low density receptor-related protein } \\ \text { EGF } & \text { epidermal growth factor } \\ \text { TGF } \beta & \text { transforming growth factor } \beta \\ \text { LAP } & \text { latency associated peptide }\end{array}$

\section{Introduction}

The TSPs are a family of five extracellular calcium-binding proteins. The functions of TSP-1 have been explored in the greatest detail because it was the first to be discovered and because it is readily purified from human blood platelets (Chen et al. 2000). TSP-1 and -2 are similar to extracellular matrix proteins in that they contain distinct domains that bind to proteoglycans (PGs), membrane proteins, including integrins, and other matrix proteins. TSP-1 is expressed at the cell surface during tissue genesis and remodeling. In the extracellular matrix (ECM) and at the cell surface, TSP-1 and -2 are transiently expressed to orchestrate the intermolecular interactions that are essential for dynamic remodeling of tissues. These properties have led to the proposal that TSP-1 and -2, as well as the other proteins described in this compendium of reviews, comprise a new class of proteins, 
designated matricellular proteins (Bornstein and Sage 2002). Matricellular proteins participate in development and tissue remodeling by regulating the structure and function of the ECM and by modulating cellular phenotype. To accomplish these functions, matricellular proteins interact with a wide array of proteins and PGs. Whereas TSP-1 and -2 are archetypal matricellular proteins, it is now clear that other TSPs have the properties of a matricellular protein. TSP-5 (also known as cartilage oligomeric matrix protein or COMP) affects cellular attachment and survival, and interacts with multiple ECM molecules (Briggs and Chapman 2002; Budde et al. 2005; Chen et al. 2007). TSP5 also influences chondrogenesis and affects ECM structure through interactions with collagens, matrilins, and PGs (Chen et al. 2007; Halasz et al. 2007; Mann et al. 2004). The characterization of TSP-3 and -4 are in their infancy and their complete credentials as matricellular proteins have not been fully established.

In general, ECM proteins interact with each other to form a structural framework that supports tissue organization and cellular processes. The matrix provides environmental and positional cues that regulate cellular behavior. This is a dynamic process in that the cell synthesizes proteins and PGs that comprise the matrix and proteases that degrade it. Thus, physiological and pathophysiological stimuli that modulate cellular phenotype lead to matrix remodeling. Most ECM proteins probably participate in this dynamic reciprocity to some extent; however, matricellular proteins are principally involved in these remodeling processes. In the tumor microenvironment, TSP-1 regulates angiogenesis, matrix metalloproteinase 9 (MMP9) activity, the activation of transforming growth factor $\beta$ (TGF $\beta$ ) and metastasis (Lawler and Detmar 2004). In the growth plate microenvironment, TSP-5 orchestrates collagen fibril formation, extracellular matrix organization, and chondrocyte survival and differentiation. In this review, we will summarize the interactions of TSPs with ECM molecules and discuss their role in ECM formation and degradation.

\section{Early studies}

In 1982, Lahav et al. (1982) performed experiments that were designed to identify proteins that mediate the interaction of platelets with ECM. In these studies, either fibronectin or type I collagen was treated with a chemical crosslinker and adsorbed to glass cover slips. The cover slips were then incubated with either platelets or the supernatant from collagen-treated platelets. With this method, TSP-1 was found to be associated with either fibronectin- or type I collagen-coated cover slips. Whereas the bound protein was simply referred to as TSP because these studies preceded the identification of other family members, it has been shown that platelets only contain TSP-1 (Kyriakides et al. 2003). A direct interaction of fibronectin with TSP-1 that is secreted from endothelial cells was also demonstrated (Lahav et al. 1982).

In 1984, Mumby et al. (1984) observed a specific interaction of TSP-1 with type V collagen but not with type I, type III or type IV collagen. Interactions with laminin, fibronectin, fibrinogen, von Willebrand factor (vWF), Factor IXa, and Factor Xa were also detected in this study. A subsequent study by Galvin et al. (1987) confirmed the interaction of type V collagen with TSP-1 and showed that binding is considerably higher in the absence of calcium ions. The authors performed the binding assays with proteolytic fragments of TSP-1 in order to map the type $\mathrm{V}$ collagen binding site. The binding of TSP-1 to type $\mathrm{V}$ collagen was reported to be mediated by a 70,000 dalton proteolytic fragment of TSP-1 that includes the procollagen homology region, the TSRs and the type 2 repeats. Like most large ECM glycoproteins, the TSPs are composed of multiple distinct structural and functional domains that reflect exon shuffling during evolution (Fig. 1) (Lawler and Hynes 1986). TSP-1, -2, -3 and -4 have $\mathrm{N}$-terminal $\beta$-sandwich domains that mediate heparin binding (Tan et al. 2006) and references therein). The Nterminal domain is followed by a region where the chains are assembled into trimers (TSP-1 and -2, Subgroup A) or pentamers (TSP-3, -4 and -5 , Subgroup B). In TSP-1 and 2 , three type 1 repeats (TSRs) follow a region of homology with procollagen that probably facilitates subunit assembly. Many of the activities of TSP-1, including the inhibition of angiogenesis and the activation of TGF $\beta$, are mediated by the TSRs (Tucker 2004; Good et al. 1990; Schultz-Cherry and Murphy-Ullrich 1993). In TSP-1 and -2, the TSRs are followed by three epidermal growth factor (EGF)-like repeats. The Subgroup B TSPs lack the region of homology with procollagen and the three TSRs, and have four EGF-

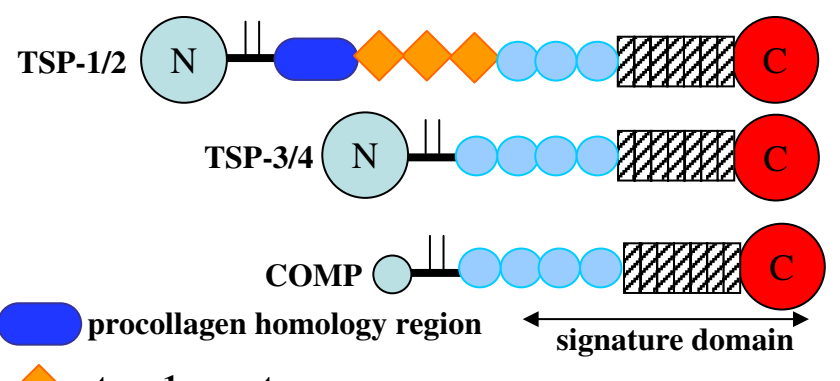

type 1 repeats

type 2 repeats

Fig. 1 Schematic representation of the members of the TSP gene family. TSP-1 and -2 (Subgroup A) have equivalent domain structures and are trimeric. TSP-3, -4 and -5 (Subgroup B) assemble into pentamers. The vertical lines represent the disulfide bonds that connect the subunits 
like repeats instead of three. In all TSPs, the EGF repeats are followed by thirteen type 3 repeats that are a series of contiguous calcium binding sites and a $\mathrm{C}$-terminal $\beta$ sandwich (Carlson et al. 2005; Kvansakul et al. 2004; Tan et al. 2009). The latter EGF repeats, the type 3 repeats and the $\mathrm{C}$-terminal $\beta$-sandwich fold into a structure that has multiple interactions between the various types of sequence. This region has been designated the "signature domain" because it is highly conserved and characteristic of the TSPs (Carlson et al. 2005). Below, we will summarize the interactions of ECM proteins with each TSP individually and conclude with a discussion of common features and properties.

\section{Thrombospondin-1}

TSP-1 affects ECM structure and function through direct interactions and through indirect effects on other components that are secreted by the cell (Fig. 2). It affects the composition of the extracellular matrix by activating TGF $\beta$, which induces the synthesis of collagens and other matrix molecules (Wynn 2008). Fibrinogen was one of the first proteins to be shown to bind TSP-1 and the formation of the fibrin clot was the first demonstration that the presence of TSP-1 can affect matrix assembly (Bale and Mosher 1986a, b). Fibrin fibrils form more rapidly in the presence of TSP-1, and are thinner and more numerous than those formed in the absence of TSP-1. TSP-1 is covalently bound to fibrin clots through its TSRs by the activity of Factor XIIIa (Bale and Mosher 1986b; Panetti et al. 1999). TSP-1 can also become incorporated into ECM through an interaction with fibronectin (Lahav et al. 1982; Leung and Nachman 1982; Sottile and Hocking 2002). Fibronectin reportedly binds to the N-terminal domain of TSP-1 and to a proteolytic fragment that contains the EGF-like repeats and the TSRs (Dardik and Lahav 1999). The existence of two sites is consistent with data that indicates that the

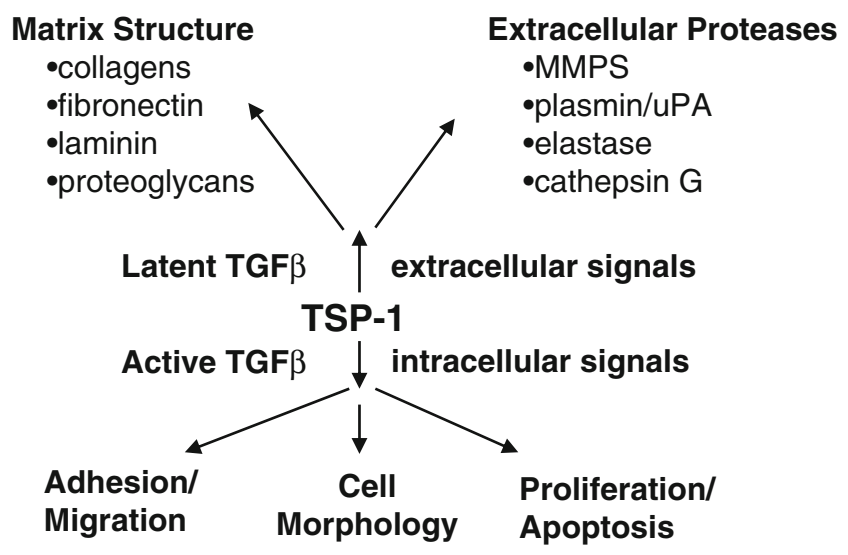

Fig. 2 The matricellular functions of TSP-1 binding of fibronectin to TSP-1 is a two step process in which binding to one site induces a conformational change that renders the second site available (Dardik and Lahav 1999). The fibronectin-induced conformational change in TSP-1 promotes the interaction of TSP- 1 with $\alpha 3 \beta 1$ integrin (Rodriguez-Manzaneque et al. 2001). The binding of fibronectin to TSP-1 also stabilizes calcium-dependent structures and protects TSP-1 from degradation (Dardik and Lahav 1999).

TSP-1 is the only member of the TSP gene family with the ability to activate TGF $\beta$ (Young and Murphy-Ullrich $2004 a, b)$. The activation of TGF $\beta$ by TSP-1 has been shown to involve the second TSR and the amino acid sequence RFK has been reported to be essential (Young and Murphy-Ullrich 2004a, b). The RFK sequence is thought to compete with the sequence RKPK in active TGF $\beta$ for a binding site in the latency-associated protein (LAP). The binding of TSP-1 to the LAP presumably induces a conformational change that allows the active domain to bind to its receptor. Whereas the precise molecular mechanism for the activation of TGF $\beta$ by TSP-1 is uncertain, the significance of the effect has been demonstrated in vivo in a wide range of biological processes including fibrosis, neoplasia and the response to injury. Cutaneous wounds in TSP-1-null mice exhibited decreased total and active TGF $\beta$, decreased collagen content, decreased macrophage recruitment and delayed wound closure (Agah et al. 2002). Consistent with this result, anti-sense knockdown of TSP-1 decreases reepithelialization of wounds (DiPietro et al. 1996). By contrast, wounds in TSP-2-null mice heal more rapidly (Kyriakides et al. 1999). Wound healing in TSP-1 and -2 double-null mice is similar to the TSP-1-null mice indicating that the initial deposition of TSP-1 by platelets determines the temporal pattern of healing (Agah et al. 2002). More effective recovery is also observed in TSP-1-null mice in the cutaneous flap assay where tissue ischemia is rate limiting (Isenberg et al. 2007). Consistent with the observation that TSP-1 antagonizes tissue perfusion that is induced by nitric oxide, TSP-1-null mice, as well as CD47-null mice, display decreased necrosis and improved healing in this model. A similar effect is seen with full thickness skin grafts where those done onto wild-type mice fail, while the majority of those performed with TSP-1-null or CD47-null mice survive (Isenberg et al. 2008).

Repair of heart tissue after myocardial infarction involves the formation of granualtion tissue and the recruitment of inflammatory cells (Frangogiannis et al. 2005). Effective cardiac repair requires that the immune response be limited spatially to the infracted myocardium. The interface between the injured tissue and the surrounding unaffected myocardium is referred to as the boarder zone. TSP-1 is expressed in the border zone where it is 
thought to limit the expansion of fibrosis in healing myocardial infarcts (Chatila et al. 2007). TSP-1-null mice exhibit increased inflammation and expansion of granulation tissue into adjacent, non-infarcted areas. Thus, activation of TGF $\beta$ by TSP-1 may suppress the recruitment of inflammatory cells and secretion of cytokines in wild-type hearts (Frangogiannis et al. 2005; Sezaki et al. 2005).

It has recently been reported that TSP-1 is deposited in matrix-associated puncta below $\mathrm{C} 2 \mathrm{C} 12$ skeletal myoblasts grown in monolayer culture (Adams et al. 2008). The incorporation into puncta is a general property of TSPs in that Drosophila TSP and the subgroup B member, TSP-5, are also located to these structures when they are expressed on COS-7 cells. The incorporation of TSP-1 into these puncta is diminished when the RGD sequence or the potential metal ion-dependent adhesion site (MIDAS) motif is mutated. Whereas $\beta 1$ integrin does enhance the inclusion of TSP-1 into puncta, it is not essential. How these puncta relate to 3-D matrix assembly in vivo remains to be determined (see below).

Proteoglycans are a major constituent of the ECM and also function as cell-surface receptors for a wide range of molecules. In most cases, the binding of TSPs to PGs is mediated by the glycosaminoglycan (GAGs) side chains, which, in the form of heparin, were one of the first binding partners of TSP-1 to be identified (Lawler et al. 1978). Quantitative analysis revealed that the N-terminal domain of TSP-1 binds to heparin with high affinity and crystal structures of the N-terminal domain in complex with various-sized heparins have been reported (Tan et al. 2006, 2008). These structures reveal that amino acids R42, R76 and R77 are important for heparin binding and that heparin can bridge two N-terminal domains in multiple ways. Heparin also binds to the TSRs, however, the significance of this interaction has been debated because it may not be of sufficient affinity to occur in physiological salt concentrations (Panetti et al. 1999; Yu et al. 2000). An additional site for heparin binding is present in the signature domain because a recombinant protein that lacks both the $\mathrm{N}$-terminal domain and the TSRs is retained on heparinSepharose columns (Lawler et al. 1992). In addition, TSP5, which lacks the N-terminal domain and the TSRs, binds heparin and the PG aggrecan (see below).

TSP-1 binds to heparan sulfate with affinities of 180 $262 \mathrm{nM}$, depending on the tissue source of the heparan sulfate (Herndon et al. 1999). The affinity of TSP-1 for chondroitin sulfate is $235-648 \mathrm{nM}$. Through interactions with these GAGs, TSP-1 binds to syndecan-1, -3 and -4 , perlecan, cerebroglycan, and versican (Elzie and MurphyUllrich 2004; Ferrari do Outeiro-Bernstein et al. 2002; Herndon et al. 1999; Kuznetsova et al. 2006). Proteoglycans function as receptors for TSP-1 in vitro, and TSP-1 and syndecan-1 display similar tissue distributions during murine development (Adams and Lawler 1994; Corless et al. 1992; Sun et al. 1992). The pro-angiogenic effects of the N-terminal domain of TSP-1 are mediated by syndecan- 4 on endothelial cells (Nunes et al. 2008). The interaction of TSP-1 with decorin involves the binding of amino acids 61 to 95 of TSP-1 to the dermatan side chains and the binding of a yet-to-be determined domain with the core protein (Merle et al. 1997; Winnemoller et al. 1992). Taken together, the data indicate that PGs are essential mediators of TSP-1 function.

The regulation of extracellular proteases is a critical component of ECM remodeling. TSP-1 inhibits the activity of plasmin, urokinase plasminogen activator, neutrophil elastase, and MMPs (Anonick et al. 1993; Hogg 1994; Rodriguez-Manzaneque et al. 2001). The regulation of MMPs by TSP-1 contributes to the regulation of angiogenesis and tumor cell invasion (Robinet et al. 2008; Rodriguez-Manzaneque et al. 2001). TSP-1 reportedly mediates the uptake and clearance of MMPs and thus reduces tumor cell invasion through Matrigel (Robinet et al. 2008). Through an interaction that involves the type 3 repeats, TSP-1 also binds to and inhibits the activity of cathepsin G (Hogg et al. 1993). The ability of TSP-1 to inhibit a broad spectrum of proteases may enable it to protect and stabilize newly formed ECM during tissue remodeling. The phenotype of TSP-2-null mice is due in part to its interaction with MMPs (see below).

\section{Thrombospondin-2}

The importance of TSP-2 for collagen matrix assembly can be seen in the skin and tendon of TSP-2-null mice (Kyriakides et al. 1998). The collagen fibrils in the skin are less tightly packed and less organized as compared to wild-type skin. The collagen fibrils in the tendon of the TSP-2-null mice are larger, have irregular contours and display a wider size distribution than their wild-type counterparts. Analysis of heterozygous mice reveals that these changes in collagen structure are directly correlated with gene dose. The skin of TSP-2-null mice is more elastic and the tails display greater flexibility. TSP-2-null mice also exhibit increased total bone density and cortical thickness.

The effects of TSP-2 on collagen fibrils appear to reflect both direct effects of TSP-2 on fibrillogenesis and more global effects on cellular phenotype. In the hind limb flexor tendon of TSP-2-null mice, fibroblast-associated compartments that host collagen fiber assembly are less organized than those of wild-type mice (Bornstein et al. 2004). The fibroblast membrane processes that outline these compartments are shorter and more variable in orientation. In addition, some fibroblasts appear to be undergoing apopto- 
sis, suggesting that TSP-2 promotes cell survival. The observation that TSP-2-null skin fibroblasts display defective cellular adhesion to vitronectin, type I collagen, TSP-2 and fibronectin suggests that TSP-2 also affects cellular phenotype (Bornstein and Sage 2002; Kyriakides et al. 1998). The cells that did attach displayed a significant defect in their ability to spread and organize their actin cytoskeleton. Consistent with a decreased ability of TSP-2null fibroblasts to organize the cytoskeleton, they also display a decreased ability to contract collagen gels (Maclauchlan et al. 2009). These changes in adhesion and spreading correlated with an increase in the amount of MMP2 in the conditioned media of TSP-2-null fibroblasts (Yang et al. 2001). Both TSP-1 and -2 are cleared from the extracellular environment though an interaction with low density lipoprotein receptor-related protein (LRP). This process results in the concomitant uptake and clearance of proteins that bind to TSP-1 and -2, including MMP2, MMP9 and VEGF (Greenaway et al. 2007; Maclauchlan et al. 2009). Cutaneous wounds in TSP-2-null mice exhibit increased levels of these proteins, as well as increased levels of tissue inhibitors of metalloproteinase-1 and -2 (Maclauchlan et al. 2009). The increase in extracellular MMP2 probably decreases collagen fibril stability. An effect of increased MMP2 in TSP-2-null mice is seen in the cervix where premature softening during pregnancy is associated with an up to 19-fold increase in MMP2 (Kokenyesi et al. 2004).

\section{Thrombospondin-3}

Although the characterization of TSP-3 has just begun, its similarities to other TSPs suggest that it will have many common functions with other family members. TSP-3 is principally expressed in the lung, cartilage and brain at the latter stages of development of mouse and chicken embryos (Iruela-Arispe et al. 1993; Qabar et al. 1995; Tucker et al. 1997). In cartilage, TSP-3 is expressed in the early proliferative zone and hypertrophic cartilage. TSP-3 is also expressed in osteoblasts and TSP-3-null mice display accelerated bone maturation (Hankenson et al. 2005). Chondrocytes in the growth plate of TSP-3-null mice exhibit a significant decrease in columnar alignment (Posey et al. 2008b). Whereas single knockouts of TSPs do not affect stature, mice that lack TSP-3, TSP-5 and type IX collagen display a $20 \%$ reduction in limb length (Posey et al. 2008b). These data suggest that TSP-3 contributes to ECM structure and function, however, direct binding of TSP-3 to ECM molecules has not been demonstrated. Like other TSPs, TSP-3 does bind heparin, suggesting that it is able to bind to proteoglycans (Qabar et al. 1994).

\section{Thrombospondin-4}

TSP-4 was first identified in Xenopus laevis embryos as a constituent of the myotome and skeletal muscle (Urry et al. 1998). It is also expressed in tendon where it can form mixed penatmers with TSP-5 (Hecht et al. 1998). TSP-4 expression is highly induced at the neuromuscular junction following injury and it promotes neurite outgrowth of chick retinal neurons in vitro (Arber and Caroni 1995). The effect of TSP-4 on neurite outgrowth may require co-factors or be species-dependent because purified TSP-4 does not support neurite outgrowth of embryonic murine retinal neurons (Dunkle et al. 2007). A caveat here is that the TSP-4 was not purified in the presence of calcium. This may have resulted in an irreversible change in conformation like that seen in TSP-1 because both proteins have a free sulfhydryl group in their C-terminal globular domains (Huang et al. 1997). Addition of purified TSP-4 to laminin significantly promotes neurite outgrowth as compared to substrates covered with laminin alone. TSP-4 also increased adhesion of retinal neurons to fibronectin or laminin. These results suggest that TSP-4 affects the organization of ECM proteins to enhance their activity, or activates signal transduction pathways that synergize with those that are activated by ECM proteins. A role for TSP-4 in the remodeling of the central nervous system is supported by comparative genomic studies that show that TSP-2 and -4 are more highly expressed in the brains of humans as compared to those of our close primate relative, chimpanzees (Caceres et al. 2007). \{See review by Eroglu in this issue\}

Narouz-Ott et al. (2000) have reported the results of binding assays of TSP-4 with various ECM proteins. Binding to collagen type I, II, III, and V is observed in the presence of zinc, but not calcium or EDTA. A similar pattern of binding is observed with intact TSP-4 or with the signature domain. In addition, the signature domain of intact TSP-4 is observed to associate with the N- and Cterminal ends of type I collagen by electron microscopy (Narouz-Ott et al. 2000). In a minority of cases, the TSP-4 appears to be bound to internal sites within the collagen type I molecules. In these studies, a similar distribution of bound TSP-5 molecules is observed. Whereas comparable levels of TSP-4 binding to laminin are observed in the presence and absence of cations, zinc and calcium enhance the binding of TSP-4 to fibronectin and matrilin-2 (NarouzOtt et al. 2000).

\section{Thrombospondin-5}

Whereas the function of TSP-5 is not well understood, it is clear that it interacts with other cartilage extracellular 
matrix proteins and PGs, including fibronectin, collagen I, II and IX, matrilin-3, and aggrecan (Fig. 3) (Briggs and Chapman 2002; Budde et al. 2005; Chen et al. 2007; Di Cesare et al. 2002; Thur et al. 2001). The physiological relevance of these interactions is supported by the fact that TSP-5 colocalizes with fibronectin, matrilin-3 and collagens in vivo, and by the fact that mutations in TSP-5, collagen IX, and matrilin-3 result in multiple epiphyseal dysplasia (Briggs and Chapman 2002; Hecht et al. 2005). Whereas the interaction of TSP-5 with fibronectin and various collagens involves the $\mathrm{C}$-terminal domain, like TSP-4, these interactions display different dependencies on divalent cations (Di Cesare et al. 2002; Holden et al. 2001; Rosenberg et al. 1998). Manganese and calcium support the binding of TSP-5 to fibronectin but not to collagens. Binding to collagens requires the presence of zinc or nickel. Divalent cations may affect these interactions through direct effects on the C-terminal domain or by inducing conformational changes in the type 3 repeats that, in turn, have an impact on the orientation or conformation of the C-terminal domain.

Electron microscopic studies have revealed that TSP-5 binds to discrete sites within the collagen and procollagen molecules (Rosenberg et al. 1998). The C-terminal domain of TSP-5 is observed to associate with the $300 \mathrm{~nm}$-long type I and II collagen molecules at the two ends and at sites that are approximately one-third and two-thirds of the length of the molecules. TSP-1 also reportedly binds to the ends of collagen I, III, IV and V, and to a site approximately one-third of the length from the ends of collagen V (Galvin et al. 1987). Thus, at least some of the TSP-1 and -5 binding sites in collagens fall in the triple-helical regions. The binding site for TSP-5 within collagen IX is different from that in collagen II because TSP- 5 has been reported to bind to the $\mathrm{NC} 4$ and other noncollagenous domains of collagen IX (Holden et al. 2001; Pihlajamaa et al. 2004). However, the binding site within TSP-5 for both collagens appears to be the same (Holden et al. 2001). In electron micrographs, TSP-1 molecules adopt multiple orientations with either the $\mathrm{N}$ - or $\mathrm{C}$-terminal, or the stalk region associating with collagens (Galvin et al. 1987). The

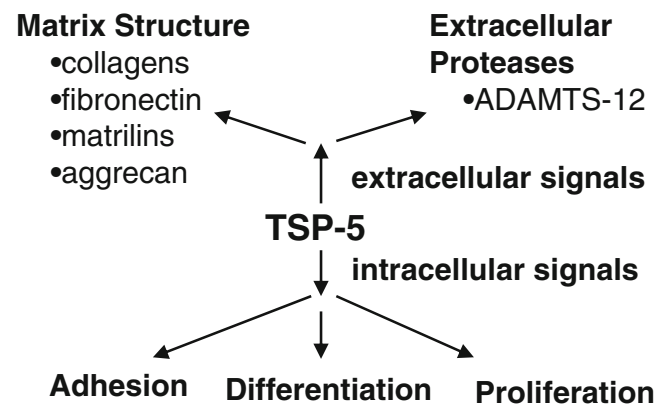

Fig. 3 The matricellular functions of TSP-5 majority of interactions appear to involve the stalk region. Taken together, it appears that the subgroup B TSPs bind to collagen through their signature domains, while TSP-1 is able to bind through this domain and others. The TSRs, which are not present in the subgroup B TSPs, may serve as one of the additional sites. The observation that the binding of TSP- 1 to collagens is inhibited by calcium suggests that calcium-dependent folding of the signature domain may limit accessibility of the collagen binding site in the stalk region when the protein is in the calcium-bound conformation (Galvin et al. 1987). This conclusion is consistent with the observation that the binding of collagen $\mathrm{V}$ to the stalk region is not affected by calcium.

TSP-5 also interacts with GAG and PG constituents of the ECM. The affinity of TSP-5 for GAGs that is commonly found in cartilage, tendon and ligament has been determined using affinity co-electrophoresis in the presence and absence of calcium (Chen et al. 2007). In the presence of calcium, TSP-5 bound to low molecular weight heparin with a comparable affinity to that of TSP-1 $(41 \mathrm{nM})$. TSP-5 also binds to chondroitin 6 sulfate (C6S), chondroitin 4 sulfate (C4S) and dermatan sulfate (DS), while the binding to heparan sulfate was weaker and no binding to keratan sulfate was observed. Depletion of calcium reduced the affinity of TSP-5 for heparin to a $\mathrm{K}_{\mathrm{d}}$ of $477 \mathrm{nM}$ and an interaction with C6S could no longer be detected. Aggrecan is a major secreted chondroitin sulfate PG of cartilage. Soluble C4S and C6S, as well as heparin, are able to inhibit the binding of TSP-5 to aggrecan, with the level of inhibition paralleling the affinity of TSP-5 for these GAGs (Chen et al. 2007). These studies also demonstrated that a recombinant version of the signature domain of TSP-5 binds to aggrecan.

The ability to partner with multiple matrix constituents raises the possibility that TSP-5 participates in ECM assembly by forming a molecular bridge between the various matrix components or between the matrix and the cell surface (Budde et al. 2005). A role for TSP-5 in matrix remodeling is suggested by the observations that its expression is up-regulated by subjecting chondrocytes to long-term cyclic compression (Giannoni et al. 2003). Furthermore, over-expression of wild-type TSP-5 results in less organized collagen fibrils with a greater number of electron dense nodules (Dinser et al. 2002). These data suggest that TSP-5 is involved in collagen fibril organization. Indeed, pentameric TSP-5 has recently been shown to promote collagen fibrillogenesis(Halasz et al. 2007). These results are consistent with the observation that the growth plate is disorganized in the TSP-5-null mice (Posey et al. 2008b). The ability of TSP-5 to bridge ECM proteins, including type II collagen may be important for the formation of inclusions of endoplasmic reticulum in pseudoachondroplasia (Briggs and Chapman 2002; Hecht 
et al. 2005). The inclusions that form in affected individuals eventually fill the cytoplasm of the chrondrocytes, causing them to die. Deconvolution microscopy indicates that ECM begins to assemble in the ER (Merritt et al. 2007).

Abnormal matrix structure is observed when some naturally-occurring mutant forms of TSP-5 are expressed (Dinser et al. 2002; Schmitz et al. 2006). This effect is observed whether or not the mutant protein is retained in the ER. When mutants that are retained in the ER are overexpressed, reduced TSP-5 secretion may underlie the observed defects in matrix structure (Schmitz et al. 2006); however, for mutants that are not retained, the defect is likely to arise from altered interaction with cell surface and matrix proteins. The decrease in growth-plate organization that has been observed in the TSP-5-null mice may reflect a defect in matrix structure (Posey et al. 2008a).

\section{Potential common features of the interaction of ECM proteins with TSPs}

Whereas TSP-1 may have multiple collagen-binding sites, the subgroup B TSPs appear to bind collagen through their signature domains. The specific molecular interactions that mediate the association of collagens with TSPs are unknown, although recent structural studies identify an intriguing possibility. Crystal structures of the C-terminal regions of TSP-1, -2 and -5 reveal that there are three highly conserved $\mathrm{Ca}^{2+}$ binding sites (designated $\mathrm{Ca} 1, \mathrm{Ca} 2$ and $\mathrm{Ca} 3$ ) in the $\mathrm{C}$-terminal domain of these molecules, in addition to the predicted $\mathrm{Ca}^{2+}$ binding sites that are associated with the type 3 repeats. The first two $\mathrm{Ca}^{2+}$ ions $(\mathrm{Ca} 1$ and $\mathrm{Ca} 2)$ in the $\mathrm{C}$-terminal domain are only about 3.8-4.3 $\AA$ apart, forming an extended $\mathrm{Ca}^{2+}$ binding site. The third $\mathrm{Ca}^{2+}$ is about $8.4 \AA$ to $9.7 \AA$ away from the first two. The triangular geometry of these three $\mathrm{Ca}^{2+}$ ions seems to be quite conserved and all three $\mathrm{Ca}^{2+}$ sites are solvent-exposed (Fig. 4). Interestingly, the molecular contacts that involve $\mathrm{Ca} 1$ and $\mathrm{Ca} 2$ in the crystals of TSP- 1 and -5 suggest that the extended $\mathrm{Ca}^{2+}$-binding site may act like a metal ion-dependent adhesion site (MIDAS) (Kvansakul et al. 2004; Tan et al. 2009). In the structure of the Cterminal region of TSP-1, an aspartic acid (D825) from type 3 repeat $7 \mathrm{C}$ of a neighboring symmetry-related molecule interacts with the extended $\mathrm{Ca}^{2+}$ binding site in such a way that it completes the coordination of $\mathrm{Ca}$ and contributes one coordinate to $\mathrm{Ca} 2$ (Kvansakul et al. 2004). The aspartic acid ligand also forms multiple hydrogen bonds with residues forming or surrounding the extended $\mathrm{Ca}^{2+}$-binding site, including a residue (Q954) from a $\beta 1 \beta 2$ loop. This loop is not a part of the extended $\mathrm{Ca}^{2+}$-binding site. However, in TSP-1, the $\beta 1 \_\beta 2$ loop itself comprises an extra $\mathrm{Ca}^{2+}$ binding site on the $\mathrm{C}$-terminal domain (Kvansakul et al.

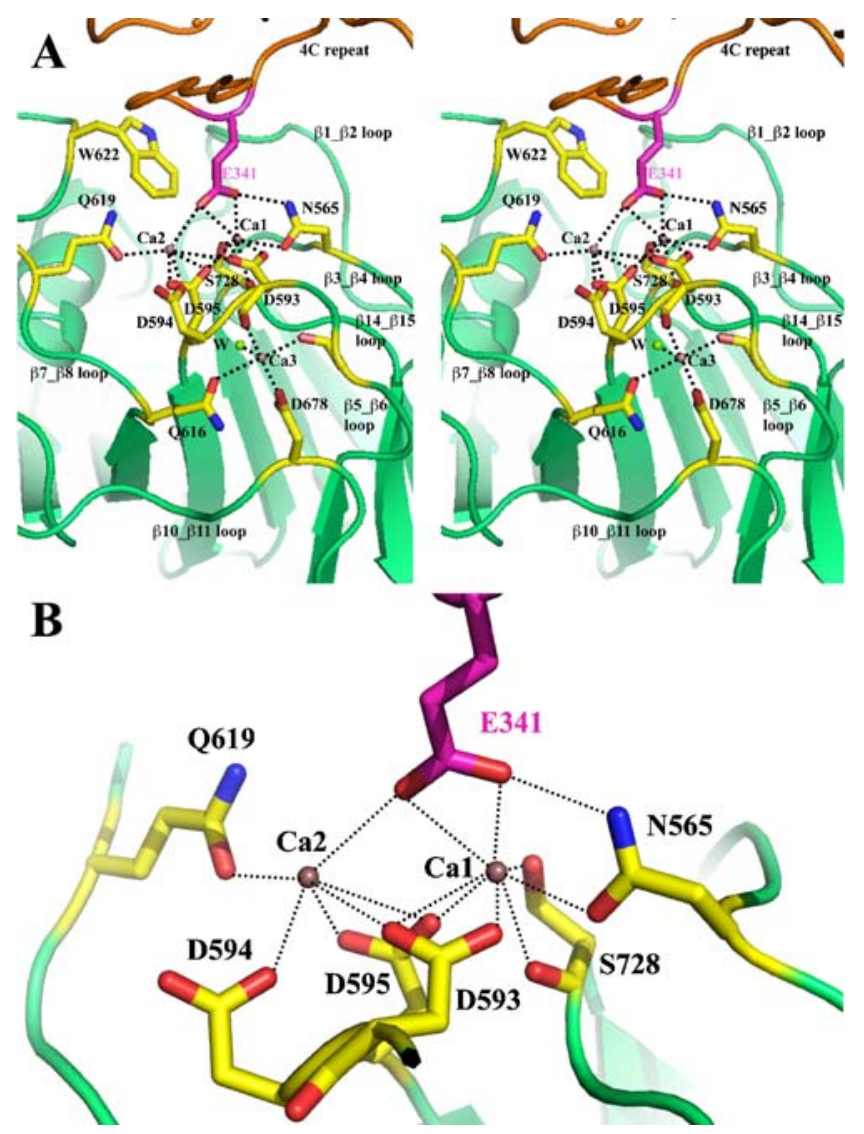

Fig. 4 The binding of a mimetic ligand to the MIDAS-like motif of TSP-5. a Stereo ribbon drawing of the MIDAS-like motif of TSP-5 and its interaction with a mimetic ligand (E341) from type 3 repeat $4 \mathrm{C}$ of a neighboring molecule in the crystal structure. The MIDAS-like motif is on the top of $\mathrm{C}$-terminal domain and includes an extended $\mathrm{Ca}^{2+}$ (purple spheres) binding site involving $\mathrm{Ca} 1$ and $\mathrm{Ca} 2$ of $\mathrm{C}$-terminal domain. The $\mathrm{Ca} 3$ binding site is next to the extended $\mathrm{Ca}$ site, sharing an aspartic acid residue, D593. The adjacent Ca site may form an ADMIDAS-like motif as discussed in the text. A water molecule is shown as a green sphere and marked with a W. There are two other water molecules that are required to complete $\mathrm{Ca} 2$ and $\mathrm{Ca} 3$ coordinates and are not resolved in the TSP-5 structure due to low-resolution limit. b A zoom in view of MIDAS-like motif and the interacting glutamic acid. For clarity, the view is slightly different from that in Fig. 4a. The figure was prepared using the program PyMOL (http://www.PyMOL.org)

2004). Type 3 repeat $7 \mathrm{C}$ is at the beginning of the recombinant TSP-1 construct and the repeat has significant conformational differences from that in its highly conserved counterparts, TSP-2 and -5 .

The MIDAS-like motif of TSP-5 is shown in Fig. 4 and is discussed in a previous publication (Tan et al. 2009). In the crystal structure of the signature domain of TSP-5, molecular packing again shows intermolecular interactions that involve the MIDAS-like motif. In TSP-5, the sidechain of a glutamic acid from type 3 repeat 4C (E341) interacts with the extended $\mathrm{Ca}^{2+}$-binding site of a neighboring TSP-5 molecule. It seems that the glutamic acid primarily interacts with $\mathrm{Ca} 1$ and weakly binds to $\mathrm{Ca} 2$, 
having less contact with other protein atoms from the MIDAS-like motif, as compared to the D825 of TSP-1. There are no residues from the $\beta 1 \_\beta 2$ loop of TSP-5 that interact with the E341. The $\beta 1 \_\beta 2$ loop of TSP-5, as well as that in TSP-2, does not host a $\mathrm{Ca}^{2+}$-binding site (Carlson et al. 2005; Tan et al. 2009). The fact that all three TSP-5 molecules in one asymmetric unit are independently involved in such MIDAS-mediated interactions with their neighboring molecules shows a strong tendency for the MIDAS-like motif of TSP-5 to participate in glutamic acidcentered ligand binding. The primary sequences that form the extended $\mathrm{Ca}^{2+}$-binding site are highly conserved (T592DDDYAG in TSP-5) throughout the members of the TSP gene family and the MIDAS-like motif is expected to also exist in the two other family members, TSP-3 and -4 , for which structures are not currently available. All TSP family members may utilize the MIDAS-like motif in ligand binding by accepting a glutamic acid or even an aspartic acid from the ligand. This type of interaction is similar to the way in which collagen II binds to the integrin $\alpha 2 \beta 1$ and ICAMs bind to $\alpha \mathrm{L} \beta 2$ (Arnaout et al. 2002; Emsley et al. 2000; Shimaoka et al. 2003; Song et al. 2005). Since all five human TSPs have been reported to bind to collagen, it is possible that TSP/collagen binding is in part through the highly conserved MIDAS-like motif in the Cterminal globular domain.

The potential MIDAS-like motifs of the TSPs have both similarities and differences with those of integrins. These sites in the TSPs are unique in that they contain two calcium ions that interact with the acidic amino acid from the ligand, as opposed to the single divalent metal ion $\left(\mathrm{Mg}^{2+}, \mathrm{Mn}^{2+}\right.$, $\mathrm{Co}^{2+}$ etc.) (Arnaout et al. 2002; Luo et al. 2007). In contrast, $\mathrm{Ca}^{2+}$ seems to be a low affinity, or even inhibitory cation for the MIDAS site in the integrins. The extended cation binding sites in the MIDAS-like motifs of the TSPs may permit variation of ligand-binding modes, such that an aspartic acid mediates the interaction in TSP-1 and a glutamic acid mediates the interaction in TSP-5. In the integrin $\beta$ subunits, an additional cation-binding site that is occupied by a $\mathrm{Ca}^{2+}$ is designated as ADMIDAS for adjacent to MIDAS. This cation binding site is reported to regulate the activity of the MIDAS (Valdramidou et al. 2008). A similar site, Ca3 is found in the TSPs close to their MIDAS-like motifs (Tan et al. 2009). It is currently unknown whether $\mathrm{Ca} 3$ of the TSPs can function like the ADMIDAS of the integrins to regulate ligand docking.

\section{Conclusions and future directions}

Matricellular proteins act at the interface of the ECM and the cell surface to facilitate tissue remodeling. They provide environmental cues to cells, which, in turn, modulate the composition of proteins that that are secreted into the extracellular environment. TSPs participate in the formation, organization and turnover of the ECM. Their multimeric structure enables them to act as bridges between fibers or between the cell surface and fibrillar structures. For TSP-2 and -5 , experimental evidence that these proteins affect collagen fibril assembly has already been obtained. At the cell surface, early collagen fibrils are associated with extensions of the plasma membrane called fibripositors (Canty and Kadler 2005). Once secreted, collagen fibrils enlarge through lateral and end-to-end associations. It has been proposed that five collagen molecules associate to form a 3-D fiber (Perumal et al. 2008; Herr and Farndale 2009). Thus, the pentameric TSPs may facilitate collagen fibrillogenesis by co-localizing five collagen molecules (Halasz et al. 2007). $\beta 1$ integrins have also been reported to participate in collagen fibrillogenesis (Anonick et al. 1993; Halasz et al. 2007). Since collagen matrix assembly is initiated at the cell surface, it will be interesting to determine whether or not integrin binding activity of the TSPs is needed for matrix assembly (Bornstein et al. 2004; Canty and Kadler 2005). Thus, a defect in the ability of TSPs to engage integrins may affect collagen fibril formation in fibripositors. The incorporation of TSP-1 into the puncta that form below cells is diminished when the RGD sequence is mutated to VGD (Adams et al. 2008). It is possible that these puncta are a 2-D manifestation of sites for matrix assembly at the cell surface.

The structural studies suggest that the potential MIDASlike motif of the TSPs functions as a binding site for collagen. Biochemical and structural studies are needed to establish that the binding of TSPs to collagen does indeed parallel the interaction of collagen to the MIDAS of integrins. These studies are best performed with subgroup B TSPs because experiments performed with TSP-1 suggest that the subgroup A TSPs have multiple collagen binding sites. The importance of the MIDAS can be established by showing that site-directed mutagenesis of the calcium-binding residues within the MIDAS affects collagen binding. In addition, collagen peptide libraries could be used to ask if a glutamic acid residue is required for TSP binding. A determination of the molecular basis for the zinc dependence of TSP binding to collagen should also be a goal of future studies. Does zinc occupy the MIDAS of the TSPs and directly participate in collagen binding or does it regulate the activity of the MIDAS? Further studies are also needed to determine if the ADMIDAS of the TSPs is functional. It will also be interesting to determine if zinc affects collagen binding by occupying this site.

Taken together, the data indicate that the TSPs serve as extracellular adapter molecules that are able to bind to a wide range of ECM proteins. The diversity of these interactions enables the TSPs to guide tissue remodeling in a variety of 
settings. The importance of TSP-1 in this context is underscored by the fact that it is rapidly deposited by platelets at the sites of tissue damage. An association of TSPs with collagens has been known for over 25 years, and the disruption of the TSP-2 gene in mice leads to collagen fibril abnormalities. Recent biochemical studies have shown that TSP-5 promotes collagen fibril assembly and structural studies have shed new light on how the TSPs may interact with collagen (Kvansakul et al. 2004; Tan et al. 2009).

Acknowledgments The authors thank Sami Lawler and Raji Bhat for help in preparing the manuscript. This work was supported by NIH grants HL049081 and CA130895.

Open Access This article is distributed under the terms of the Creative Commons Attribution Noncommercial License which permits any noncommercial use, distribution, and reproduction in any medium, provided the original author(s) and source are credited.

\section{References}

Adams JC, Lawler J (1994) Cell-type specific adhesive interactions of skeletal myoblasts with thrombospondin-1. Mol Biol Cell 5:423437

Adams JC, Bentley AA, Kvansakul M, Hatherley D, Hohenester E (2008) Extracellular matrix retention of thrombospondin 1 is controlled by its conserved C-terminal region. J Cell Sci 121:784-795

Agah A, Kyriakides TR, Lawler J, Bornstein P (2002) The lack of thrombospondin-1 (TSP1) dictates the course of wound healing in double-TSP1/TSP2-null mice. Am J Pathol 161:831-839

Anonick PK, Yoo JK, Webb DJ, Gonias SL (1993) Characterization of the antiplasmin activity of human thrombospondin-1 in solution. Biochem J 289(Pt 3):903-909

Arber S, Caroni P (1995) Thrombospondin-4, an extracellular matrix protein expressed in the developing and adult nervous system promotes neurite outgrowth. J Cell Biol 131:1083-1094

Arnaout MA, Goodman SL, Xiong JP (2002) Coming to grips with integrin binding to ligands. Curr Opin Cell Biol 14:641-651

Bale MD, Mosher DF (1986a) Thrombospondin is a substrate for blood coagulation factor XIIIa. Biochemistry 25:5667-5673

Bale MD, Mosher DF (1986b) Effects of thrombospondin on fibrin polymerization and structure. J Biol Chem 261:862-868

Bornstein P, Sage EH (2002) Matricellular proteins: extracellular modulators of cell function. Curr Opin Cell Biol 14:608-616

Bornstein P, Agah A, Kyriakides TR (2004) The role of thrombospondins 1 and 2 in the regulation of cell-matrix interactions, collagen fibril formation, and the response to injury. Int $\mathrm{J}$ Biochem Cell Biol 36:1115-1125

Briggs MD, Chapman KL (2002) Pseudoachondroplasia and multiple epiphyseal dysplasia: mutation review, molecular interactions, and genotype to phenotype correlations. Hum Mutat 19:465-478

Budde B, Blumbach K, Ylostalo J, Zaucke F, Ehlen HW, Wagener R, Ala-Kokko L, Paulsson M, Bruckner P, Grassel S (2005) Altered integration of matrilin-3 into cartilage extracellular matrix in the absence of collagen IX. Mol Cell Biol 25:10465-10478

Caceres M, Suwyn C, Maddox M, Thomas JW, Preuss TM (2007) Increased cortical expression of two synaptogenic thrombospondins in human brain evolution. Cereb Cortex 17:2312-2321

Canty EG, Kadler KE (2005) Procollagen trafficking, processing and fibrillogenesis. J Cell Sci 118:1341-1353
Carlson CB, Bernstein DA, Annis DS, Misenheimer TM, Hannah BL, Mosher DF, Keck JL (2005) Structure of the calcium-rich signature domain of human thrombospondin-2. Nat Struct Mol Biol 12:910-914

Chatila K, Ren G, Xia Y, Huebener P, Bujak M, Frangogiannis NG (2007) The role of the thrombospondins in healing myocardial infarcts. Cardiovasc Hematol Agents Med Chem 5:21-27

Chen H, Herndon ME, Lawler J (2000) The cell biology of thrombospondin-1. Matrix Biol 19:597-614

Chen FH, Herndon ME, Patel N, Hecht JT, Tuan RS, Lawler J (2007) Interaction of cartilage oligomeric matrix protein/thrombospondin 5 with aggrecan. J Biol Chem 282:24591-24598

Corless CL, Mendoza A, Collins T, Lawler J (1992) Colocalization of thrombospondin and syndecan during murine development. Dev Dyn 193:346-358

Dardik R, Lahav J (1999) Functional changes in the conformation of thrombospondin-1 during complexation with fibronectin or heparin. Exp Cell Res 248:407-414

Di Cesare PE, Chen FS, Moergelin M, Carlson CS, Leslie MP, Perris R, Fang C (2002) Matrix-matrix interaction of cartilage oligomeric matrix protein and fibronectin. Matrix Biol 21:461-470

Dinser R, Zaucke F, Kreppel F, Hultenby K, Kochanek S, Paulsson M, Maurer P (2002) Pseudoachondroplasia is caused through both intra- and extracellular pathogenic pathways. J Clin Invest 110:505-513

DiPietro LA, Nissen NN, Gamelli RL, Koch AE, Pyle JM, Polverini PJ (1996) Thrombospondin 1 synthesis and function in wound repair. Am J Pathol 148:1851-1860

Dunkle ET, Zaucke F, Clegg DO (2007) Thrombospondin-4 and matrix three-dimensionality in axon outgrowth and adhesion in the developing retina. Exp Eye Res 84:707-717

Elzie CA, Murphy-Ullrich JE (2004) The N-terminus of thrombospondin: the domain stands apart. Int $\mathrm{J}$ Biochem Cell Biol 36:1090-1101

Emsley J, Knight CG, Farndale RW, Barnes MJ, Liddington RC (2000) Structural basis of collagen recognition by integrin alpha2beta1. Cell 101:47-56

Ferrari do Outeiro-Bernstein MA, Nunes SS, Andrade AC, Alves TR, Legrand C, Morandi V (2002) A recombinant NH(2)-terminal heparin-binding domain of the adhesive glycoprotein, thrombospondin-1, promotes endothelial tube formation and cell survival: a possible role for syndecan-4 proteoglycan. Matrix Biol 21:311-324

Frangogiannis NG, Ren G, Dewald O, Zymek P, Haudek S, Koerting A, Winkelmann K, Michael LH, Lawler J, Entman ML (2005) Critical role of endogenous thrombospondin-1 in preventing expansion of healing myocardial infarcts. Circulation 111:2935-2942

Galvin NJ, Vance PM, Dixit VM, Fink B, Frazier WA (1987) Interaction of human thrombospondin with types I-V collagen: direct binding and electron microscopy. J Cell Biol 104:1413-1422

Giannoni P, Siegrist M, Hunziker EB, Wong M (2003) The mechanosensitivity of cartilage oligomeric matrix protein (COMP). Biorheology 40:101-109

Good DJ, Polverini PJ, Rastinejad F, LeBeau MM, Lemons RS, Frazier WA, Bouck NP (1990) A tumor suppressor-dependent inhibitor of angiogenesis is immunologically and functionally indistinguishable from a fragment of thrombospondin. Proc Natl Acad Sci USA 87:6624-6628

Greenaway J, Lawler J, Moorehead R, Bornstein P, Lamarre J, Petrik J (2007) Thrombospondin-1 inhibits VEGF levels in the ovary directly by binding and internalization via the low density lipoprotein receptor-related protein-1 (LRP-1). J Cell Physiol 210:807-818

Halasz K, Kassner A, Morgelin M, Heinegard D (2007) COMP acts as a catalyst in collagen fibrillogenesis. J Biol Chem 282:3116631173 
Hankenson KD, Hormuzdi SG, Meganck JA, Bornstein P (2005) Mice with a disruption of the thrombospondin 3 gene differ in geometric and biomechanical properties of bone and have accelerated development of the femoral head. Mol Cell Biol 25:5599-5606

Hecht JT, Deere M, Putnam E, Cole W, Vertel B, Chen H, Lawler J (1998) Characterization of cartilage oligomeric matrix protein (COMP) in human normal and pseudoachondroplasia musculoskeletal tissues. Matrix Biol 17:269-278

Hecht JT, Hayes E, Haynes R, Cole WG (2005) COMP mutations, chondrocyte function and cartilage matrix. Matrix Biol 23:525-533

Herndon ME, Stipp CS, Lander AD (1999) Interactions of neural glycosaminoglycans and proteoglycans with protein ligands: assessment of selectivity, heterogeneity and the participation of core proteins in binding. Glycobiology 9:143-155

Herr AB, Farndale RW (2009) Structural insights into the interaction between platelet receptors and fibrillar collagen. J Biol Chem 284:19781-19785

Hogg PJ (1994) Thrombospondin 1 as an enzyme inhibitor. Thromb Haemost 72:787-792

Hogg PJ, Owensby DA, Chesterman CN (1993) Thrombospondin 1 is a tight-binding competitive inhibitor of neutrophil cathepsin G. Determination of the kinetic mechanism of inhibition and localization of cathepsin $\mathrm{G}$ binding to the thrombospondin 1 type 3 repeats. J Biol Chem 268:21811-21818

Holden P, Meadows RS, Chapman KL, Grant ME, Kadler KE, Briggs MD (2001) Cartilage oligomeric matrix protein interacts with type IX collagen, and disruptions to these interactions identify a pathogenetic mechanism in a bone dysplasia family. J Biol Chem 276:6046-6055

Huang EM, Detwiler TC, Milev Y, Essex DW (1997) Thiol-disulfide isomerization in thrombospondin: effects of conformation and protein disulfide isomerase. Blood 89:3205-3212

Iruela-Arispe ML, Liska DJ, Sage EH, Bornstein P (1993) Differential expression of thrombospondin 1, 2, and 3 during murine development. Dev Dyn 197:40-56

Isenberg JS, Hyodo F, Pappan LK, Abu-Asab M, Tsokos M, Krishna MC, Frazier WA, Roberts DD (2007) Blocking thrombospondin1/CD47 signaling alleviates deleterious effects of aging on tissue responses to ischemia. Arterioscler Thromb Vasc Biol 27:25822588

Isenberg JS, Pappan LK, Romeo MJ, Abu-Asab M, Tsokos M, Wink DA, Frazier WA, Roberts DD (2008) Blockade of thrombospondin-1-CD47 interactions prevents necrosis of full thickness skin grafts. Ann Surg 247:180-190

Kokenyesi R, Armstrong LC, Agah A, Artal R, Bornstein P (2004) Thrombospondin 2 deficiency in pregnant mice results in premature softening of the uterine cervix. Biol Reprod 70:385390

Kuznetsova SA, Issa P, Perruccio EM, Zeng B, Sipes JM, Ward Y, Seyfried NT, Fielder HL, Day AJ, Wight TN, Roberts DD (2006) Versican-thrombospondin-1 binding in vitro and colocalization in microfibrils induced by inflammation on vascular smooth muscle cells. J Cell Sci 119:4499-4509

Kvansakul M, Adams JC, Hohenester E (2004) Structure of a thrombospondin C-terminal fragment reveals a novel calcium core in the type 3 repeats. Embo J 23:1223-1233

Kyriakides TR, Zhu YH, Smith LT, Bain SD, Yang Z, Lin MT, Danielson KG, Iozzo RV, LaMarca M, McKinney CE, Ginns EI, Bornstein P (1998) Mice that lack thrombospondin 2 display connective tissue abnormalities that are associated with disordered collagen fibrillogenesis, an increased vascular density, and a bleeding diathesis. J Cell Biol 140:419-430

Kyriakides TR, Tam JW, Bornstein P (1999) Accelerated wound healing in mice with a disruption of the thrombospondin 2 gene. J Invest Dermatol 113:782-787
Kyriakides TR, Rojnuckarin P, Reidy MA, Hankenson KD, Papayannopoulou T, Kaushansky K, Bornstein P (2003) Megakaryocytes require thrombospondin-2 for normal platelet formation and function. Blood 101:3915-3923

Lahav J, Schwartz MA, Hynes RO (1982) Analysis of platelet adhesion with a radioactive chemical crosslinking reagent: interaction of thrombospondin with fibronectin and collagen. Cell 31:253-262

Lawler J, Hynes RO (1986) The structure of human thrombospondin, an adhesive glycoprotein with multiple calcium-binding sites and homologies with several different proteins. J Cell Biol 103:16351648

Lawler J, Detmar M (2004) Tumor progression: the effects of thrombospondin-1 and -2. Int J Biochem Cell Biol 36:1038-1045

Lawler JW, Slayter HS, Coligan JE (1978) Isolation and characterization of a high molecular weight glycoprotein from human blood platelets. J Biol Chem 253:8609-8616

Lawler J, Ferro P, Duquette M (1992) Expression and mutagenesis of thrombospondin. Biochemistry 31:1173-1180

Leung LL, Nachman RL (1982) Complex formation of platelet thrombospondin with fibrinogen. J Clin Invest 70:542-549

Luo BH, Carman CV, Springer TA (2007) Structural basis of integrin regulation and signaling. Annu Rev Immunol 25:619647

Maclauchlan S, Skokos EA, Agah A, Zeng J, Tian W, Davidson JM, Bornstein P, Kyriakides TR (2009) Enhanced angiogenesis and reduced contraction in thrombospondin-2-null wounds is associated with increased levels of matrix metalloproteinases-2 and -9 , and soluble VEGF. J Histochem Cytochem 57:301-313

Mann HH, Ozbek S, Engel J, Paulsson M, Wagener R (2004) Interactions between the cartilage oligomeric matrix protein and matrilins. Implications for matrix assembly and the pathogenesis of chondrodysplasias. J Biol Chem 279:25294-25298

Merle B, Malaval L, Lawler J, Delmas P, Clezardin P (1997) Decorin inhibits cell attachment to thrombospondin-1 by binding to a KKTR-dependent cell adhesive site present within the N-terminal domain of thrombospondin-1. J Cell Biochem 67:75-83

Merritt TM, Bick R, Poindexter BJ, Alcorn JL, Hecht JT (2007) Unique matrix structure in the rough endoplasmic reticulum cisternae of pseudoachondroplasia chondrocytes. Am J Pathol 170:293-300

Mumby SM, Raugi GJ, Bornstein P (1984) Interactions of thrombospondin with extracellular matrix proteins: selective binding to type V collagen. J Cell Biol 98:646-652

Narouz-Ott L, Maurer P, Nitsche DP, Smyth N, Paulsson M (2000) Thrombospondin-4 binds specifically to both collagenous and non-collagenous extracellular matrix proteins via its $\mathrm{C}$-terminal domains. J Biol Chem 275:37110-37117

Nunes SS, Outeiro-Bernstein MA, Juliano L, Vardiero F, Nader HB, Woods A, Legrand C, Morandi V (2008) Syndecan-4 contributes to endothelial tubulogenesis through interactions with two motifs inside the pro-angiogenic N-terminal domain of thrombospondin1. J Cell Physiol 214:828-837

Panetti TS, Kudryk BJ, Mosher DF (1999) Interaction of recombinant procollagen and properdin modules of thrombospondin-1 with heparin and fibrinogen/fibrin. J Biol Chem 274:430-437

Perumal S, Antipova O, Orgel JPRO (2008) Collagen fibril architecture, domain organization, and triple-helical conformation governs its proteolysis. Proc Natl Acad Sci USA 105: 2824-2829

Pihlajamaa T, Lankinen H, Ylostalo J, Valmu L, Jaalinoja J, Zaucke F, Spitznagel L, Gosling S, Puustinen A, Morgelin M, Peranen J, Maurer P, Ala-Kokko L, Kilpelainen I (2004) Characterization of recombinant amino-terminal NC4 domain of human collagen IX: interaction with glycosaminoglycans and cartilage oligomeric matrix protein. J Biol Chem 279:24265-24273 
Posey KL, Yang Y, Veerisetty AC, Sharan SK, Hecht JT (2008a) Model systems for studying skeletal dysplasias caused by TSP-5/ COMP mutations. Cell Mol Life Sci 65:687-699

Posey KL, Hankenson K, Veerisetty AC, Bornstein P, Lawler J, Hecht JT (2008b) Skeletal abnormalities in mice lacking extracellular matrix proteins, thrombospondin-1, thrombospondin-3, thrombospondin-5, and type IX collagen. Am J Pathol 172:1664-1674

Qabar AN, Lin Z, Wolf FW, O'Shea KS, Lawler J, Dixit VM (1994) Thrombospondin 3 is a developmentally regulated heparin binding protein. J Biol Chem 269:1262-1269

Qabar A, Derick L, Lawler J, Dixit V (1995) Thrombospondin 3 is a pentameric molecule held together by interchain disulfide linkage involving two cysteine residues. J Biol Chem 270:12725-12729

Robinet A, Emonard H, Banyai L, Laronze JY, Patthy L, Hornebeck W, Bellon G (2008) Collagen-binding domains of gelatinase A and thrombospondin-derived peptides impede endocytic clearance of active gelatinase A and promote HT1080 fibrosarcoma cell invasion. Life Sci 82:376-382

Rodriguez-Manzaneque JC, Lane TF, Ortega MA, Hynes RO, Lawler J, Iruela-Arispe ML (2001) Thrombospondin-1 suppresses spontaneous tumor growth and inhibits activation of matrix metalloproteinase-9 and mobilization of vascular endothelial growth factor. Proc Natl Acad Sci U S A 98:12485-12490

Rosenberg K, Olsson H, Morgelin M, Heinegard D (1998) Cartilage oligomeric matrix protein shows high affinity zinc-dependent interaction with triple helical collagen. J Biol Chem 273:2039720403

Schmitz M, Becker A, Schmitz A, Weirich C, Paulsson M, Zaucke F, Dinser R (2006) Disruption of extracellular matrix structure may cause pseudoachondroplasia phenotypes in the absence of impaired cartilage oligomeric matrix protein secretion. J Biol Chem 281:32587-32595

Sezaki S, Hirohata S, Iwabu A, Nakamura K, Toeda K, Miyoshi T, Yamawaki H, Demircan K, Kusachi S, Shiratori Y, Ninomiya Y (2005) Thrombospondin-1 is induced in rat myocardial infarction and its induction is accelerated by ischemia/reperfusion. Exp Biol Med (Maywood) 230:621-630

Shimaoka M, Xiao T, Liu JH, Yang Y, Dong Y, Jun CD, McCormack A, Zhang R, Joachimiak A, Takagi J, Wang JH, Springer TA (2003) Structures of the alpha L I domain and its complex with ICAM-1 reveal a shape-shifting pathway for integrin regulation. Cell 112:99-111

Schultz-Cherry S, Murphy-Ullrich JE (1993) Thrombospondin causes activation of latent transforming growth factor-beta secreted by endothelial cells by a novel mechanism. J Cell Biol 122:923-932

Song G, Yang Y, Liu JH, Casasnovas JM, Shimaoka M, Springer TA, Wang JH (2005) An atomic resolution view of ICAM recognition in a complex between the binding domains of ICAM-3 and integrin alphaLbeta2. Proc Natl Acad Sci U S A 102:3366- 3371

Sottile J, Hocking DC (2002) Fibronectin polymerization regulates the composition and stability of extracellular matrix fibrils and cellmatrix adhesions. Mol Biol Cell 13:3546-3559

Sun X, Kaesberg PR, Choay J, Harenberg J, Ershler WB, Mosher DF (1992) Effects of sized heparin oligosaccharide on the interac- tions of Chinese hamster ovary cell with thrombospondin. Semin Thromb Hemost 18:243-251

Tan K, Duquette M, Liu JH, Zhang R, Joachimiak A, Wang JH, Lawler J (2006) The structures of the thrombospondin-1Nterminal domain and its complex with a synthetic pentameric heparin. Structure 14:33-42

Tan K, Duquette M, Liu JH, Shanmugasundaram K, Joachimiak A, Gallagher JT, Rigby AC, Wang JH, Lawler J (2008) Heparininduced cis- and trans-dimerization modes of the thrombospondin1N-terminal domain. J Biol Chem 283:3932-3941

Tan K, Duquette M, Joachimiak A, Lawler J (2009) The crystal structure of the signature domain of cartilage oligomeric matrix protein: implications for collagen, glycosaminoglycan and integrin binding. Faseb J. 23:2490-2501

Thur J, Rosenberg K, Nitsche DP, Pihlajamaa T, Ala-Kokko L, Heinegard D, Paulsson M, Maurer P (2001) Mutations in cartilage oligomeric matrix protein causing pseudoachondroplasia and multiple epiphyseal dysplasia affect binding of calcium and collagen I, II, and IX. J Biol Chem 276:60836092

Tucker RP (2004) The thrombospondin type 1 repeat superfamily. Int J Biochem Cell Biol 36:969-974

Tucker RP, Hagios C, Chiquet-Ehrismann R, Lawler J (1997) In situ localization of thrombospondin-1 and thrombospondin-3 transcripts in the avian embryo. Dev Dyn 208:326-337

Urry LA, Whittaker CA, Duquette M, Lawler J, DeSimone DW (1998) Thrombospondins in early Xenopus embryos: dynamic patterns of expression suggest diverse roles in nervous system, notochord, and muscle development. Dev Dyn 211:390-407

Valdramidou D, Humphries MJ, Mould AP (2008) Distinct roles of beta1 metal ion-dependent adhesion site (MIDAS), adjacent to MIDAS (ADMIDAS), and ligand-associated metal-binding site (LIMBS) cation-binding sites in ligand recognition by integrin alpha2beta1. J Biol Chem 283:32704-32714

Winnemoller M, Schon P, Vischer P, Kresse H (1992) Interactions between thrombospondin and the small proteoglycan decorin: interference with cell attachment. Eur J Cell Biol 59:47-55

Wynn TA (2008) Cellular and molecular mechanisms of fibrosis. J Pathol 214:199-210

Yang Z, Strickland DK, Bornstein P (2001) Extracellular matrix metalloproteinase 2 levels are regulated by the low density lipoprotein-related scavenger receptor and thrombospondin 2. J Biol Chem 276:8403-8408

Young GD, Murphy-Ullrich JE (2004a) Molecular interactions that confer latency to transforming growth factor-beta. J Biol Chem 279:38032-38039

Young GD, Murphy-Ullrich JE (2004b) The tryptophan-rich motifs of the thrombospondin type 1 repeats bind VLAL motifs in the latent transforming growth factor-beta complex. J Biol Chem 279:47633-47642

Yu H, Tyrrell D, Cashel J, Guo NH, Vogel T, Sipes JM, Lam L, Fillit HM, Hartman J, Mendelovitz S, Panel A, Roberts DD (2000) Specificities of heparin-binding sites from the amino-terminus and type 1 repeats of thrombospondin-1. Arch Biochem Biophys $374: 13-23$ 